PROCEEDINGS OF THE

AMERICAN MATHEMATICAL SOCIETY

Volume 130, Number 11, Pages 3151-3158

S 0002-9939(02)06440-7

Article electronically published on April 17, 2002

\title{
ASYMPTOTIC BEHAVIOR OF REDUCTION NUMBERS
}

\author{
LÊ TUÂN HOA
}

(Communicated by Wolmer V. Vasconcelos)

\begin{abstract}
It is shown that the reduction number and the big reduction number of $S / I^{n}$ are linear functions of $n$ for all large $n$. Here $I$ is a homogeneous ideal of a polynomial ring $S$.
\end{abstract}

\section{INTRODUCTION}

Let $I$ be an ideal of a Noetherian $\operatorname{ring} A$. An ideal $J \subseteq I$ is called a reduction of $I$ if $I^{n+1}=J I^{n}$ for some $n \geq 0$. The minimal number among such $n$ is called the reduction number of $I$ w.r.t. $J$ and is denoted by $r_{J}(I)$. We call a reduction $J$ of $I$ a minimal reduction if it does not properly contain another reduction of $I$ (see $[\mathrm{NR}]$ ). Assume that $I$ has minimal reductions (which is always the case in our consideration below). We call

$$
r(I)=\min \left\{r_{J}(I) ; J \text { is a minimal reduction of } I\right\}
$$

the reduction number of $I$ and

$$
\operatorname{br}(I)=\max \left\{r_{J}(I) ; J \text { is a minimal reduction of } I\right\}
$$

the big reduction number of $I$. The reduction number of $I$ was introduced by Sally (see $[\underline{\mathrm{S}}]$ ) and the big reduction number was recently considered by Vasconcelos [V2]. It is clear that $r(I)$ is finite, while the finiteness of $b r(I)$ easily follows from [T1] (see also [S, Remark on p. 237] and [V2 for other proofs). If $A$ is a local ring or a standard graded ring over a field and $I=\mathfrak{m}$ is the maximal (homogeneous) ideal of $A$, then we simply set

$$
r(A)=r(\mathfrak{m}) \text { and } \operatorname{br}(A)=b r(\mathfrak{m}) .
$$

In this paper we study the asymptotic values of $r\left(S / I^{n}\right)$ and $b r\left(S / I^{n}\right)$, where $I$ is a homogeneous ideal of a polynomial ring $S=k\left[x_{1}, \ldots, x_{s}\right]$. This problem was raised in a recent study of the asymptotic behavior of the CastelnuovoMumford regularity in [HHT]. It was shown in [HHT, Theorem 1.5] that both limits $\lim _{n \rightarrow \infty} \frac{r\left(S / I^{n}\right)}{n}$ and $\lim _{n \rightarrow \infty} \frac{b r\left(S / I^{n}\right)}{n}$ do exist. This fact also holds if $I$ is an ideal in a local ring $A$. However, in the local case an example constructed there shows that $r\left(A / I^{n}\right)$ and $b r\left(A / I^{n}\right)$ need not be linear functions of $n$ for large $n$. On

Received by the editors March 23, 2001 and, in revised form, June 5, 2001.

1991 Mathematics Subject Classification. Primary 13A15.

Key words and phrases. Reduction number, Castelnuovo-Mumford regularity.

The author was supported by the National Basic Research Program (Vietnam) and University of Essen (Germany). 
the other hand, it was recently shown in [CHT, Theorem 3.1] and [K, Theorem 5] that the Castelnuovo-Mumford regularity $\operatorname{reg}\left(S / I^{n}\right)$ is in fact a linear function of $n$ for large $n$. Since there are close relations between the reduction number and the Castelnuovo-Mumford regularity (see [T1] and [T2]), it should be expected that in the polynomial case the reduction number of $S / I^{n}$ is eventually a linear function of $n$. In fact we can show that not only the minimal value $r\left(S / I^{n}\right)$ among all $r_{J}\left(S / I^{n}\right)$ but also the maximal value $\operatorname{br}\left(S / I^{n}\right)$ among them have this property. This is the content of our main result:

Theorem 1.1. Let $S=k\left[x_{1}, \ldots, x_{s}\right]$ be a polynomial ring over an infinite field and $I$ a proper homogeneous ideal of $S$ of height $h$. Then there exist integers $b, B \geq 0$ and $c, C \geq-1$ such that

(i) $r\left(S / I^{n}\right)=b n+c$ for all $n \gg 0$ and $b n-1 \leq r\left(S / I^{n}\right) \leq b(n+h-1)-h$ for all $n$.

(ii) $b r\left(S / I^{n}\right)=B n+C$ for all $n \gg 0$ and $B n-1 \leq b r\left(S / I^{n}\right) \leq B(n+h-1)-h$ for all $n$.

If $I$ in addition is generated by forms of the same degree $D$, then $b=B=D$.

The proof of this theorem will be found in Section 2. Examples will be given to show that these integers $b, B, c$ and $C$ may be different. In Section 3 , as a byproduct of our approach, we will improve Vasconcelos' bound for the big reduction number of an algebra given in [V1].

\section{Proof of the Main Result}

From now on let $S=k\left[x_{1}, \ldots, x_{s}\right]$ be a polynomial ring over an infinite field $k$ and $I$ a proper nonzero homogeneous ideal of $S$. Let $\mathfrak{m}=\left(x_{1}, \ldots, x_{s}\right)$. By abuse of terminology we will say that a homogeneous ideal $\mathfrak{q} \subseteq \mathfrak{m}$ is a (minimal) reduction of $S / I$ if its image $\overline{\mathfrak{q}}$ is a (minimal) reduction of the image $\overline{\mathfrak{m}}$ of $\mathfrak{m}$ in $S / I$. We also denote $r_{\overline{\mathfrak{q}}}(\overline{\mathfrak{m}})$ simply by $r_{\mathfrak{q}}(S / I)$.

It is useful to observe that if $I \subseteq J$ are two homogeneous ideals, then any reduction $\mathfrak{q}$ of $S / I$ is also a reduction of $S / J$ and $r_{\mathfrak{q}}(S / J) \leq r_{\mathfrak{q}}(S / I)$. For any $n \geq 1, \mathfrak{q}$ is a (minimal) reduction of $S / I$ if and only if it is a (minimal) reduction of $S / I^{n}$. The following auxiliary result provides an upper bound for reduction numbers:

Lemma 2.1. Let $D(I)$ denote the maximal degree of elements in a minimal set of homogeneous generators of $I$. Then for any minimal reduction $\mathfrak{q}$ of $S / I$ we have

$$
r_{\mathfrak{q}}\left(S / I^{n}\right) \leq r_{\mathfrak{q}}(S / I)+(n-1) D(I) .
$$

Proof. It suffices to show that $r_{\mathfrak{q}}\left(S / I^{n}\right) \leq r_{\mathfrak{q}}\left(S / I^{n-1}\right)+D(I)$. Let $r=r_{\mathfrak{q}}\left(S / I^{n-1}\right)$ and $D=D(I)$. Since $I$ is generated by elements of degrees at most $D$, it is easy to see that $(\mathfrak{q}+I) \cap \mathfrak{m}^{r+1+D} \subseteq \mathfrak{q}+I \mathfrak{m}^{r+1}$. By the assumption, $\mathfrak{m}^{r+1} \subseteq \mathfrak{q}+I^{n-1} \subseteq \mathfrak{q}+I$. Hence we have

$$
\begin{aligned}
\mathfrak{m}^{r+1+D} & \subseteq(\mathfrak{q}+I) \cap \mathfrak{m}^{r+1+D} \subseteq \mathfrak{q}+I \mathfrak{m}^{r+1} \\
& \subseteq \mathfrak{q}+I\left(\mathfrak{q}+I^{n-1}\right) \subseteq \mathfrak{q}+I^{n} .
\end{aligned}
$$

For a graded $S$-module $M$, let

$$
a(M)=\sup \left\{i ;[M]_{i} \neq 0\right\} .
$$


Note that $a(M)<\infty$ if $M$ is Artinian (we set $a(0):=-\infty$ ). Then, for a reduction $\mathfrak{q}$ of $S / I$ we have

$$
r_{\mathfrak{q}}(S / I)=a(S / \mathfrak{q}+I) .
$$

Further, let $\left\{f_{1}, \ldots, f_{u}\right\}$ be a minimal homogeneous generating set of $I$. Denote by $F$ the set of elements $f_{i}, 1 \leq i \leq u$, such that for all positive integers $n$ we have $f_{i}^{n} \notin \mathfrak{m} I^{n}$. Let $p$ be the largest degree of polynomials in $F$. Since an element $f_{j}$ with the smallest degree clearly belongs to $F, p$ is well defined and $p \leq D(I)$. This construction and a part of the proof of the following result follow $[\mathrm{K}$ Proposition $4]$.

Lemma 2.2. Assume that $I \subset S$ is an $\mathfrak{m}$-primary homogeneous ideal. With the above notation we have

$$
p n-1 \leq r\left(S / I^{n}\right)=b r\left(S / I^{n}\right) \leq p(n+s-1)-s .
$$

Proof. Since $S / I^{n}$ is an Artinian ring, $r\left(S / I^{n}\right)=b r\left(S / I^{n}\right)=a\left(S / I^{n}\right)$. Note that for any $f \in F$ of degree $p$ and for any $n, f^{n}$ is an element of degree $p n$ in a minimal generating set of $I^{n}$. Hence, there must be a homogeneous polynomial of degree $p n-1$ which is not in $I^{n}$, i.e.

$$
r\left(S / I^{n}\right)=a\left(S / I^{n}\right) \geq p n-1 .
$$

Further, let $J=(F)$ and $K$ be the ideal generated by $\left\{f_{1}, \ldots, f_{u}\right\} \backslash F$. From the definition of $F$ it then follows that there exists $n_{0}$ such that $K^{n} \subseteq \mathfrak{m} I^{n}$ for all $n \geq n_{0}$. Then $I^{n}=(J+K)^{n}=J(J+K)^{n-1}+K^{n} \subseteq J I^{n-1}+\mathfrak{m} I^{n}$. By Nakayama's lemma it follows that $I^{n}=J I^{n-1}$. Hence $J$ is an $\mathfrak{m}$-primary ideal. Since $J$ is generated by elements of degree at most $p$, one can choose a regular sequence $y_{1}, \ldots, y_{s}$ of degree $p$ in $J$. We have

$$
\begin{aligned}
\operatorname{br}\left(S / I^{n}\right) & =r_{0}\left(S / I^{n}\right) \leq r_{0}\left(S / J^{n}\right) \leq r_{0}\left(S /\left(y_{1}, \ldots, y_{s}\right)^{n}\right) \\
& \leq r_{0}\left(S /\left(y_{1}, \ldots, y_{s}\right)\right)+(n-1) p, \quad(\text { by Lemma } 2.1) \\
& =a\left(S /\left(y_{1}, \ldots, y_{s}\right)\right)+(n-1) p \\
& =s(p-1)+(n-1) p=p(n+s-1)-s .
\end{aligned}
$$

Lemma 2.3. Let $\mathfrak{q}$ be a minimal reduction of $S / I$. Let $p \geq 0$ and $q$ be some integers. Assume that $r_{\mathfrak{q}}\left(S / I^{n}\right) \leq p n+q$ for some $n \geq q+2$. Then $r_{\mathfrak{q}}\left(S / I^{m}\right) \leq$ $p m+q$ for all $m \geq n$.

Proof. It suffices to prove the statement for $m=n+1$. First we show that

$$
\left(\mathfrak{q}+I^{n}\right) \cap \mathfrak{m}^{p n+q+1} \subseteq \mathfrak{q}+I \mathfrak{m}^{p(n-1)+q+1} .
$$

Considering the quotient ring $S / \mathfrak{q}$ we may assume that $\mathfrak{q}=0$. Of course we are working now with a general graded ring. Let $f=f_{1} \ldots f_{n} \in I^{n} \cap \mathfrak{m}^{p n+q+1}$, where $f_{1}, \ldots, f_{n} \in I$ and $\operatorname{deg} f_{1} \leq \cdots \leq \operatorname{deg} f_{n}$. Let $f^{\prime}=f_{2} \ldots f_{n}$. If $\operatorname{deg} f_{1} \leq p$, then $\operatorname{deg} f^{\prime} \geq \operatorname{deg} f-p \geq p(n-1)+q+1$. If $\operatorname{deg} f_{1} \geq p+1$, then $\operatorname{deg} f^{\prime} \geq(n-1)(p+1) \geq$ $p(n-1)+q+1$ (because $n \geq q+2)$. In both cases we have $f=f_{1} f^{\prime} \in I \mathfrak{m}^{p(n-1)+q+1}$. Thus (1) is proven. 
On the other hand, by the assumption we get $\mathfrak{m}^{p n+q+1} \subseteq \mathfrak{q}+I^{n}$. Hence, from (11) it follows that

$$
\begin{aligned}
\mathfrak{m}^{p(n+1)+q+1} & =\mathfrak{m}^{p}\left(\left(\mathfrak{q}+I^{n}\right) \cap \mathfrak{m}^{p n+q+1}\right) \\
& \subseteq \mathfrak{m}^{p}\left(\mathfrak{q}+I \mathfrak{m}^{p(n-1)+q+1}\right) \\
& \subseteq \mathfrak{q}+I \mathfrak{m}^{p n+q+1} \\
& \subseteq \mathfrak{q}+I\left(\mathfrak{q}+I^{n}\right) \\
& =\mathfrak{q}+I^{n+1} .
\end{aligned}
$$

Remark. The author is grateful to the referee for remarks which simplify the proof of Lemmas 2.1 and 2.3

Now we are able to prove the main result stated in the introduction.

Proof of Theorem 1.1. First we prove the statement for the reduction number. Let $d=\operatorname{dim} S / I$ and $\mathfrak{q} \subseteq \mathfrak{m}$ be a minimal reduction of $S / I$. We can assume that $\mathfrak{q}$ is generated by $d$ independent linear forms $\left(z_{s-d+1}, \ldots, z_{s}\right)$. Choose other $(s-d)$ linear forms $z_{1}, \ldots, z_{s-d}$ such that $S=k\left[z_{1}, \ldots, z_{s}\right]$. Let $S^{\prime}=S / \mathfrak{q} \cong k\left[z_{1}, \ldots, z_{s-d}\right]$ and $I^{\prime}$ the image of $I$. Since $S /\left(\mathfrak{q}+I^{n}\right) \cong S^{\prime} / I^{\prime n}, r_{\mathfrak{q}}\left(S / I^{n}\right)=a\left(S^{\prime} / I^{\prime n}\right)=r_{0}\left(S^{\prime} / I^{\prime n}\right)$, by Lemma 2.2 we can find a positive integer $b(\mathfrak{q})$ such that

$$
\begin{aligned}
b(\mathfrak{q}) n-1 \leq r_{\mathfrak{q}}\left(S / I^{n}\right)=r_{0}\left(S^{\prime} / I^{\prime n}\right) & \leq b(\mathfrak{q})(n+s-d-1)-(s-d) \\
& =b(\mathfrak{q})(n+h-1)-h .
\end{aligned}
$$

From (2) it follows that $b(\mathfrak{q})=\lim _{n \rightarrow \infty} \frac{r_{\mathfrak{q}}\left(S / I^{n}\right)}{n}$, and hence it is uniquely defined (for each $\mathfrak{q})$.

Choose a minimal reduction $J$ of $S / I$ such that

$$
b(J)=b:=\min \{b(\mathfrak{q}) ; \mathfrak{q} \text { is a minimal reduction of } S / I\} .
$$

Fix an integer $n$. Assume that $r\left(S / I^{n}\right)=r_{\mathfrak{q}}\left(S / I^{n}\right)$ for a minimal reduction $\mathfrak{q}$. Then by (21)

$$
r\left(S / I^{n}\right)=r_{\mathfrak{q}}\left(S / I^{n}\right) \geq b(\mathfrak{q}) n-1 \geq b n-1 .
$$

This proves the lower bound of the first statement.

On the other hand, by (2) we also have

$$
r\left(S / I^{n}\right) \leq r_{J}\left(S / I^{n}\right) \leq b(J)(n+h-1)-h=b(n+h-1)-h,
$$

which provides the upper bound of the first statement.

Since both lower and upper bounds of $r\left(S / I^{n}\right)$ are linear functions of $n$ with the same leading coefficient, there is the least integer $c \geq-1$ such that

$$
r\left(S / I^{n}\right) \leq b n+c, \text { for all } n \gg 0 .
$$

We show that we eventually have the equality in (3). Assume that there is $n \geq c+1$ such that $r\left(S / I^{n}\right) \leq b n+c-1$. Choose a minimal reduction $\mathfrak{q}$ of $S / I^{n}$ such that $r\left(S / I^{n}\right)=r_{\mathfrak{q}}\left(S / I^{n}\right)$. Since $r_{\mathfrak{q}}\left(S / I^{n}\right) \leq b n+c-1$ and $n \geq c+1=(c-1)+2$, by Lemma 2.3 we would have $r\left(S / I^{m}\right) \leq r_{\mathfrak{q}}\left(S / I^{m}\right) \leq b m+c-1$ for all $m \geq n$, which contradicts the minimality of $c$ satisfying (3). This finishes the proof of the first statement.

The proof for the big reduction number is similar. The only difference is that we should choose $B$ to be the maximal among $b(\mathfrak{q})$. 
If $I$ is an ideal generated by forms of the same degree $D$, then by the definition of $b(\mathfrak{q})$ it follows that all $b(\mathfrak{q})=D$. Hence $b=B=D$. The proof of Theorem 1.1 is completed.

As a consequence of Theorem 1.1 we get

Corollary 2.4. Let $y_{i}=a_{i 1} x_{1}+\cdots+a_{i s} x_{s}, i=1, \ldots, d=\operatorname{dim} S / I$, and $a_{i j} \in k$. If $a_{i j}$ are chosen generically, then $\mathfrak{q}=\left(y_{1}, \ldots, y_{d}\right)$ is a minimal reduction of $S / I$ with $r_{\mathfrak{q}}\left(S / I^{n}\right)=r\left(S / I^{n}\right)$ for all $n$.

Proof. Assume that $r\left(S / I^{n}\right)=b n+c$ for all $n \geq n_{0}>c+2$, where $b, c$ are given by Theorem 1.1. Since $k$ is infinite, by [T2, Lemma 4.2] a generic choice of $a_{i j}$ will ensure that $\mathfrak{q}$ is a minimal reduction of $S / I$ and $r_{\mathfrak{q}}\left(S / I^{n}\right)=r\left(S / I^{n}\right)$ for all $n \leq n_{0}$. In particular, $r_{\mathfrak{q}}\left(S / I^{n_{0}}\right)=b n_{0}+c$. By Lemma 2.3 it implies that $r\left(S / I^{n}\right) \leq r_{\mathfrak{q}}\left(S / I^{n}\right) \leq b n+c=r\left(S / I^{n}\right)$ for all $n>n_{0}$. Hence we also have $r_{\mathfrak{q}}\left(S / I^{n}\right)=r\left(S / I^{n}\right)$ for all $n>n_{0}$.

Remark. (i) The lower bounds in Theorem 1.1 for $r\left(S / I^{n}\right)$ and $b r\left(S / I^{n}\right)$ were eventually given in [HHT, Theorem 1.5]. However, the coefficients $b$ and $B$ were not explicitly determined there.

(ii) In Theorem 1.1 we always have $b \leq B \leq D=D(I)$. Thus the upper bound $\operatorname{br}\left(S / I^{n}\right) \leq B(n+h-1)-h$ strengthens the bound $\operatorname{br}(S / I) \leq D h-h$ given in $\mathrm{V2}$, Proposition 9.3.1].

(iii) In the proof of [T2, Lemma 4.2] it is shown that if we extend the field $k$ to $k(u)=k\left(u_{i j}\right)$, where $u_{i j}$ are algebraically independent, then for any ideal $I$, $r_{J_{u}}\left(S_{u} / I_{u}\right)=r(S / I)$, where $S_{u}=S \otimes k(u), I_{u}=I S_{u}$ and $J_{u}=\left(u_{i 1} x_{1}+\cdots+\right.$ $\left.u_{i s} x_{s} ; i=1, \ldots, d\right)$. Using this fact and [CHT, Theorem 3.4] one can immediately get the asymptotic behavior of $r\left(S / I^{n}\right)$ given in Theorem [1.1(i). However, this approach does not work for the big reduction number, and also could not derive Corollary 2.4 (if $k$ is not an uncountable field). We don't know whether a similar statement of Corollary 2.4 holds true for big reduction numbers, i.e. whether the big reduction numbers $\operatorname{br}\left(S / I^{n}\right), n=1,2, \ldots$ are simultaneously attained by a common minimal reduction.

(iv) Let $\overline{I^{n}}$ denote the integral closure of $I^{n}$. With a slight modification of the proof of the main theorem one can show that the reduction number $r\left(S / \overline{I^{n}}\right)$ and the big reduction number $\operatorname{br}\left(S / \overline{I^{n}}\right)$ of $S / \overline{I^{n}}$ are linear functions of $n$ for all $n \gg 0$.

It is natural to ask from which value of $n$ the reduction number $r\left(S / I^{n}\right)$ and the big reduction number $\operatorname{br}\left(S / I^{n}\right)$ become linear functions. We still cannot answer this question. However, the following consequence of the main theorem may give a hint for its solution.

Corollary 2.5. Keep the notation in Theorem 1.1 and Lemma 2.1. Let $r\left(S / I^{n}\right)=$ bn $+c_{n}$ and $\operatorname{br}\left(S / I^{n}\right)=B n+C_{n}$. Then the sequences $\left\{c_{n}\right\}$ and $\left\{C_{n}\right\}$ are monotonic non-increasing for all $n \geq(D(I)-1)(h-1)+1$.

Proof. By Theorem 1.1(i), $b n+c_{n}=r\left(S / I^{n}\right) \leq b(n+h-1)-h$. Hence $c_{n} \leq$ $b(h-1)-h$. Let $n \geq(D(I)-1)(h-1)+1$. Choose a minimal reduction $\mathfrak{q}$ of $S / I$ such that $r_{\mathfrak{q}}\left(S / I^{n}\right)=r\left(S / I^{n}\right)=b n+c_{n}$. Since

$$
n \geq(D(I)-1)(h-1)+1 \geq(b-1)(h-1)+1=b(h-1)-h+2 \geq c_{n}+2,
$$

by Lemma 2.3 we have $r_{\mathfrak{q}}\left(S / I^{n+1}\right) \leq b(n+1)+c_{n}$. This means $c_{n+1} \leq c_{n}$. The proof for $\left\{C_{n}\right\}$ is similar. 
Example 1. It was shown in $[\mathrm{H}]$ that if $I$ is an arbitrary ideal in a local ring, then for all $n \gg 0, r_{\mathfrak{q}}\left(I^{n}\right)$ does not depend on the choice of a minimal reduction $\mathfrak{q}$ of $I^{n}$, i.e. $r\left(I^{n}\right)=b r\left(I^{n}\right)$. The graded version of this result says that if $I$ is generated by forms of the same degree, then $r\left(I^{n}\right)=b r\left(I^{n}\right)$ for $n \gg 0$. This is not true for $r\left(S / I^{n}\right)$. Indeed, let

$$
I=\left(x_{1}^{6}, x_{1}^{4} x_{2}^{2}, x_{1}^{2} x_{2}^{4}, x_{2}^{6}, x_{1}^{5} x_{3}, x_{2}^{5} x_{4}\right) .
$$

Note that $x_{3}, x_{4}$ form a system of parameters of $S / I$. Hence, by [BH, Lemma 5], an arbitrary minimal reduction of $S / I$ can be written in the form $\left(x_{3}+a_{1} x_{1}+\right.$ $\left.a_{2} x_{2}, x_{4}+b_{1} x_{1}+b_{2} x_{2}\right)$. From this it follows that $r(S / I)=\operatorname{br}(S / I)=6$. Thus, in this example the reduction numbers of $S / I$ do not depend on the choice of a minimal reduction. However, we will see that $r\left(S / I^{n}\right)<b r\left(S / I^{n}\right)$ for all $n \geq 2$.

By Lemma 2.1 and Theorem 1.1 it follows that

$$
6 n-1 \leq r\left(S / I^{n}\right) \leq b r\left(S / I^{n}\right) \leq 6 n,
$$

for all $n$. Let $\mathfrak{q}_{1}=\left(x_{3}, x_{4}\right)$. Let $I_{1}$ denote the image of $I$ in $S / \mathfrak{q}_{1} \cong k\left[x_{1}, x_{2}\right]$. Since $x_{1}^{6 n-1} x_{2} \notin I_{1}^{n}=\left(x_{1}^{6}, x_{1}^{4} x_{2}^{2}, x_{1}^{2} x_{2}^{4}, x_{2}^{6}\right)^{n}$, we have $r_{\mathfrak{q}_{1}}\left(S / I^{n}\right)=a\left(k\left[x_{1}, x_{2}\right] / I_{1}^{n}\right)=6 n$. By (4) it implies that $\operatorname{br}\left(S / I^{n}\right)=6 n$.

For $n \geq 2$ let $\mathfrak{q}_{2}=\left(x_{3}-x_{2}, x_{4}-x_{1}\right)$. Denote by $I_{2}$ the image of $I$ in $S / \mathfrak{q}_{2} \cong$ $k\left[x_{1}, x_{2}\right]$. We have $I_{2}=\left(x_{1}^{6}, x_{1}^{5} x_{2}, x_{1}^{4} x_{2}^{2}, x_{1}^{2} x_{2}^{4}, x_{1} x_{2}^{5}, x_{2}^{6}\right)$. Therefore $I_{2}^{n}=\left(x_{1}, x_{2}\right)^{6 n}$ and $r_{\mathfrak{q}_{2}}\left(S / I^{n}\right)=6 n-1$. Again by (4), $r\left(S / I^{n}\right)=6 n-1$ for all $n \geq 2$.

Example 2. Consider again Example 7(1) in $[\mathrm{BH}]$. Let $I=\left(x_{1}^{a_{1}}, x_{1} x_{2}^{a_{2}-1}\right) \subset S=$ $k\left[x_{1}, x_{2}\right]$ for some $a_{1}>a_{2}$. For all $n$ we have $\operatorname{br}\left(S / I^{n}\right)=r_{\left(x_{2}\right)}\left(S / I^{n}\right)=n a_{1}-1$, while $r\left(S / I^{n}\right)=r_{\left(x_{2}-x_{1}\right)}\left(S / I^{n}\right)=n a_{2}-1$. This example shows that in Theorem $1.1 B$ can be arbitrarily larger than $b$.

\section{A BOUND FOR $\operatorname{br}(S / I)$}

The following result was proved in [HHT, Theorem 1.5]

Lemma 3.1. Let $I, J$ be homogeneous ideals of $S$ and $\mathfrak{q}$ a reduction of $S / I J$. Then

$$
r_{\mathfrak{q}}(S / I J) \leq r_{\mathfrak{q}}(S / I)+r_{\mathfrak{q}}(S / J)+1 .
$$

This lemma has an interesting application in bounding the reduction number of $S / I$. Recall that the arithmetic degree of $S / I$ is the integer

$$
\operatorname{adeg}(S / I)=\sum_{\mathfrak{p}} \operatorname{mult}_{S / I}(\mathfrak{p}) \operatorname{deg}(S / \mathfrak{p}),
$$

where $\mathfrak{p}$ runs over all associated primes of $S / I$ and $\operatorname{mult}_{S / I}(\mathfrak{p})$ is the multiplicity of $S / I$ at $\mathfrak{p}$ defined by $\ell\left(H_{\mathfrak{m}_{\mathfrak{p}}}^{0}\left((S / I)_{\mathfrak{p}}\right)\right)$. This multiplicity is in many cases much bigger than the so-called Loewy multiplicity of $M$ at $\mathfrak{p}$ defined by

$$
\operatorname{Lmult}_{S / I}(\mathfrak{p})=\ell \ell\left(H_{\mathfrak{m}_{\mathfrak{p}}}^{0}\left((S / I)_{\mathfrak{p}}\right)\right):=\min \left\{n \geq 0 ; \mathfrak{p}^{n} H_{\mathfrak{m}_{\mathfrak{p}}}^{0}\left((S / I)_{\mathfrak{p}}\right)=0\right\} .
$$

It was shown by Vasconcelos that $\operatorname{br}(S / I) \leq \operatorname{adeg}(S / I)-1$ provided $\operatorname{char}(k)=0$ (for all notations and results above, see [V1, Chapter 9]). In particular, $\operatorname{br}(S / I) \leq$ $\operatorname{deg} S / I-1$ if $I$ is a prime ideal. Here we can improve Vasconcelos' bound in the case where $S / I$ is not a domain as follows. 
Proposition 3.2. Let I be a homogeneous ideal of $S$. Assume that $\operatorname{char}(k)=0$. Then

$$
\operatorname{br}(S / I) \leq \sum_{\mathfrak{p}} \operatorname{Lmult}_{S / I}(\mathfrak{p}) \operatorname{deg}(S / \mathfrak{p})-1 .
$$

Proof. First we recall some observations on the Loewy multiplicity from [V1, Section 9.2]. Let $\mathfrak{p}$ be a homogeneous prime ideal. If $\mathfrak{q}$ is a $\mathfrak{p}$-primary ideal, then $(\sqrt{\mathfrak{q}})^{n} \subseteq \mathfrak{q}$, where $n=$ Lmult $_{S / \mathfrak{q}}(\mathfrak{p})$. Let $I=\bigcap Q_{i}$ be a primary decomposition of $I$, where $\sqrt{Q_{i}}=\mathfrak{p}_{i}$. For each maximal associated prime ideal $\mathfrak{p}_{l}$, set $J_{l}=Q_{l} \cap H_{l}$, where $H_{l}$ is the intersection of all $Q_{i}^{\prime}$ s contained in $\mathfrak{p}_{l}$ and $i \neq l$. Then for any associated prime $\mathfrak{p}$ of $J_{l}$,

$$
\operatorname{Lmult}_{S / J_{l}}(\mathfrak{p})=\operatorname{Lmult}_{S / I}(\mathfrak{p}) .
$$

From these observations, by an easy induction on the number of associated primes we can conclude that if $n_{\mathfrak{p}}=\operatorname{Lmult}_{S / I}\left(\mathfrak{p}_{i}\right)$, then

$$
\prod \mathfrak{p}^{n_{\mathfrak{p}}} \subseteq I .
$$

Let $\mathfrak{q}$ be a reduction of $S / I$. Then $\mathfrak{q}$ is also a reduction of $S / \prod \mathfrak{p}^{n_{\mathfrak{p}}}$ and of all $S / \mathfrak{p}^{n_{\mathfrak{p}}}$. Hence from (5) and Lemma 3.1 we get

$$
r_{\mathfrak{q}}(S / I)+1 \leq r_{\mathfrak{q}}\left(S / \prod \mathfrak{p}^{n_{\mathfrak{p}}}\right)+1 \leq \sum n_{\mathfrak{p}}\left(r_{\mathfrak{q}}(S / \mathfrak{p})+1\right) \leq \sum n_{\mathfrak{p}} \operatorname{deg} S / \mathfrak{p},
$$

where the last inequality follows from the above mentioned result of Vasconcelos. Therefore $\operatorname{br}(S / I) \leq \sum n_{\mathfrak{p}} \operatorname{deg} S / \mathfrak{p}-1$.

Example 3. Let $I=\left(x_{1}, \ldots, x_{p_{1}}\right)^{n_{1}} \ldots\left(x_{p_{t-1}+1}, \ldots, x_{p_{t}}\right)^{n_{t}}, p_{1}<p_{2}<\cdots<p_{t}$. This ideal is generated by monomials of degree $n_{1}+\cdots+n_{t}$. Hence, by [BH] Proposition 4], $r(S / I) \geq n_{1}+\cdots+n_{t}-1$, which equals the upper bound in the above proposition. Hence $r(S / I)=b r(S / I)=n_{1}+\cdots+n_{t}-1$ and the above upper bound is attained. In this example

$$
\operatorname{adeg}(S / I)=\left(\begin{array}{c}
n_{1}+p_{1} \\
n_{1}
\end{array}\right)+\left(\begin{array}{c}
n_{2}+p_{2}-p_{1} \\
n_{2}
\end{array}\right)+\left(\begin{array}{c}
n_{t}+p_{t}-p_{t-1} \\
n_{t}
\end{array}\right) .
$$

In some cases we can combine the results presented previously in order to compute the exact value of $r\left(S / I^{n}\right)$ :

Example 4. Assume that $r(S / I)=1$ and $I$ does not contain a linear form. Then in Theorem 1.1 we have $b \geq 2$ and $r\left(S / I^{n}\right) \geq 2 n-1$. From Lemma 3.1 it follows that $r\left(S / I^{n}\right) \leq 2 n-1$. Hence $r\left(S / I^{n}\right)=2 n-1$ for all $n$.

Example 5. Let

$$
\mathfrak{p}=\left(x_{0} x_{3}-x_{1} x_{2}, x_{1}^{b}-x_{0}^{b-a} x_{2}^{a}, x_{1}^{b-1} x_{3}-x_{0}^{b-a-1} x_{2}^{a+1}, \ldots, x_{1}^{a} x_{3}^{b-a}-x_{2}^{b}\right)
$$

be the defining prime ideal of the monomial curve $\left(t_{0}^{b+a}, t_{0}^{b} t_{1}^{a}, t_{0}^{a} t_{1}^{b}, t_{1}^{a+b}\right)$ in $\mathbf{P}^{3}$, where g.c.d. $(a, b)=1$ and $b>a>0$. It was shown in $[\mathrm{BH}$, Example 15] that $r(S / \mathfrak{p})=$ $\operatorname{br}(S / \mathfrak{p})=b-1$. Hence by Lemma 3.1 we have $\operatorname{br}\left(S / \mathfrak{p}^{n}\right) \leq b n-1$. Comparing the degree of $x_{1}$ we can see that $\left(x_{1}^{b}-x_{0}^{b-a} x_{2}^{a}\right)^{n} \notin \mathfrak{m p}^{n}$ for all $n$. This implies that $b$ exactly is the integer $b$ defined in Theorem 1.1. and so $r\left(S / \mathfrak{p}^{n}\right) \geq b n-1$. Hence $r\left(S / \mathfrak{p}^{n}\right)=b r\left(S / \mathfrak{p}^{n}\right)=b n-1$ for all $n$. 


\section{ACKNOWLEDGMENT}

Part of this paper was written while visiting the University of Essen in Summer 2000. The author would like to express his sincere thanks to the Research Group "Arithmetic and Geometry" for their support and hospitality, and to J. Herzog for many helpful discussions.

\section{REFERENCES}

[BH] H. Bresinsky and L. T. Hoa, On the reduction number of some graded algebras, Proc. Amer. Math. Soc. 127(1999), 1257-1263. MR 99h:13027

[CHT] D. Cutkosky, J. Herzog and N. V. Trung, Asymptotic behaviour of the CastelnuovoMumford regularity, Compositio math. 118(1999), 243-261. MR 2000f:13037

[HHT] J. Herzog, L. T. Hoa and N. V. Trung, Asymptotic linear bounds for the CastelnuovoMumford regularity, Trans. Amer. Math. Soc. (to appear).

$[\mathrm{H}] \quad$ L. T. Hoa, Reduction numbers and Rees algebras of powers of an ideal, Proc. Amer. Math. Soc. 119(1993), 415-422. MR 93k:13009

$[\mathrm{K}] \quad$ V. Kodiyalam, Asymptotic behaviour of Castelnuovo-Mumford regularity, Proc. Amer. Math. Soc. 128(2000), 407-411. MR 2000c:13027

[NR] D. G. Northcott and D. Rees, Reductions of ideals in local rings, Proc. Cambridge Philos. Soc. 50(1954), 145-158. MR 15:596a

[S] J. Sally, Reductions, local cohomology and Hilbert functions of local rings, Commutative Algebra (Durham 1981), London Math. Soc. Lecture Note Ser., vol. 72, Cambridge Univ. Press, Cambridge and New York, 1982, pp. 231-241. MR 84g:13037

[T1] N. V. Trung, Reduction exponent and degree bound for the defining equations of graded rings, Proc. Amer. Math. Soc. 101(1987), 229-236. MR 89i:13031

[T2] N. V. Trung, Gröbner bases, local cohomology and reduction number, Proc. Amer. Math. Soc. 129(2001), 9-18. MR 2001c:13042

[V1] W. V. Vasconcelos, Computational Methods in Commutative Algebra and Algebraic Geometry, Springer-Verlag, Berlin, 1998. MR 99c:13048

[V2] W. V. Vasconcelos, Reduction numbers of ideals, J. Algebra 216(1999), 652-664. MR 2000e: 13005

Institute of Mathematics, Box 631, Bò Hô, 10000 Hanoi, Vietnam

E-mail address: lthoa@thevinh.ncst.ac.vn 